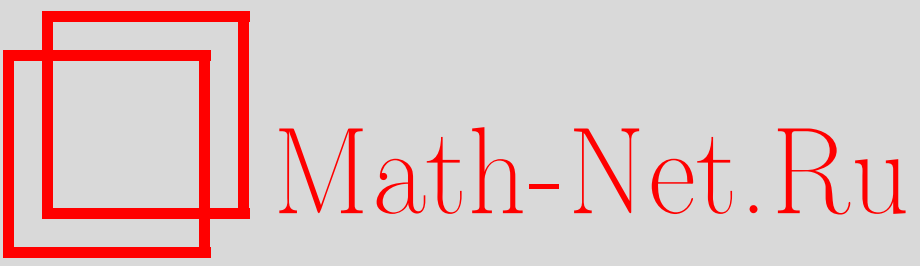

Г. В. Гаркавенко, О спектральных свойствах одного класса возмущенных операторов, Итоги науки и техн. Сер. Соврем. мат. и ее прил. Темат. обз., 2019, том 171, 57-69

DOI: https://doi.org/10.36535/0233-6723-2019-171-57-69

Использование Общероссийского математического портала Math-Net.Ru подразумевает, что вы прочитали и согласны с пользовательским соглашением

http: //www . mathnet.ru/rus/agreement

Параметры загрузки:

IP : 54.224 .135 .184

26 апреля 2023 г., 13:15:34 


\title{
О СПЕКТРАЛЬНЫХ СВОЙСТВАХ ОДНОГО КЛАССА ВОЗМУЩЕННЫХ ОПЕРАТОРОВ
}

\author{
(C) 2019 г. $\quad$ Г. В. ГАРКАВЕНКО
}

\begin{abstract}
АннотАция. При помощи метода подобных операторов исследованы спектральные свойства возмущенного оператора $A-B: D(A) \subset H \rightarrow H$, где $A-$ самосопряженный оператор, имеющий компактную резольвенту, $B$ - неограниченное возмущение. При некоторых условиях на спектр невозмущенного оператора $A$ и возмущение $B$ найдены оценки спектральных множеств возмущенного оператора. Полученные результаты применены к исследованию спектра дифференциальных операторов с периодическими краевыми условиями и негладким потенциалом.
\end{abstract}

Ключевые слова: метод подобных операторов, спектр, дифференциальный оператор.

\section{ON SPECTRAL PROPERTIES OF A CERTAIN CLASS OF PERTURBED OPERATORS}

\author{
(c) 2019 G. V. GARKAVENKO
}

\begin{abstract}
Using the method of similar operators, we examine spectral properties of the perturbed operator $A-B: D(A) \subset H \rightarrow H$, where $A$ is a self-adjoint operator with a compact resolvent and $B$ is an unbounded perturbation. Under certain conditions on the spectrum of the unperturbed operator $A$ and the perturbation $B$, we obtain estimates of the spectral sets of the perturbed operator. The results obtained are applied to the study of the spectrum of differential operators with periodic boundary conditions and a nonsmooth potential.
\end{abstract}

Keywords and phrases: method of similar operators, spectrum, differential operator.

AMS Subject Classification: 47B06, 47B37

1. Введение. Статья посвящена исследованию спектральных свойств возмущенного самосопряженного оператора с дискретным спектром, удовлетворяющим некоторым условиям, и с неограниченным возмущением. Исследование проводится с помощью метода подобных операторов. Полученные результаты для абстрактного оператора применяются к исследованию спектральных свойств дифференциальных операторов второго порядка, определяемых периодическими краевыми условиями на конечном промежутке с негладким потенциалом.

В основе проводимого исследования лежит метод подобных операторов, основные положения которого описаны в [1-3]; дальнейшее развитие метод получил в [4-8].

Для изучения спектральных свойств оператора существенно используется теорема 2.1 о подобии возмущенного оператора оператору более простой структуры. Основные результаты статьи изложены в теоремах 3.2 и 3.3 , где найдена асимптотика спектра возмущенного оператора. Полученные результаты применены в разделе 4 для исследования спектральных свойств дифференциального оператора. Асимптотика спектра описана в теореме 4.1.

Итак, будем рассматривать возмущенный оператор $A-B$, где $A$-оператор с известным спектром, удовлетворяющим некоторому условию, а $B$ - оператор возмущения. 
Пусть самосопряженный оператор $A: D(A) \subset H \rightarrow H$ имеет спектр $\sigma(A)=\bigcup_{n=1}^{\infty} \sigma_{n}$, состоящий из множеств $\sigma_{n}=\left\{\lambda_{n}\right\}$, где $\lambda_{n}$ - полупростые собственные значения. Первым предварительным условием на спектр оператора будет являться условие

$$
\operatorname{dist}\left(\lambda_{n}, \sigma_{n}(A) \backslash \lambda_{n}\right) \rightarrow \infty, \quad n \rightarrow \infty .
$$

Оператор $B$ принадлежит пространству допустимых возмущений для оператора $A$.

2. Основные положения метода подобных операторов. Приведем основные определения и теоремы, составляющие основу метода подобных операторов.

Пусть $H$ - комплексное сепарабельное гильбертово пространство, - End $H$ - банахова алгебра линейных ограниченных операторов, действующих в $H$.

Определение 2.1. Линейные операторы $A_{1}: D\left(A_{1}\right) \subset H \rightarrow H$ и $A_{2}: D\left(A_{2}\right) \subset H \rightarrow H$ называются подобными, если существует такой непрерывно обратимый оператор $U \in \operatorname{End} H$, что

$$
A_{1} U x=U A_{2} x, \quad x \in D\left(A_{2}\right), \quad U\left(D\left(A_{2}\right)\right)=D\left(A_{1}\right) .
$$

Оператор $U$ называется оператором преобразования оператора $A_{1}$ в оператор $A_{2}$.

В следующей лемме отражены спектральные свойства подобных операторов.

Лемма 2.1. Пусть оператор $A_{2}: D\left(A_{2}\right) \subset H \rightarrow H$ подобен оператору $A_{1}: D\left(A_{1}\right) \subset H \rightarrow H$ u $U \in$ End $H$ - оператор преобразования оператора $A_{1}$ в оператор $A_{2}$. Тогда справедливы следующие утверждения:

(1) $\sigma\left(A_{1}\right)=\sigma\left(A_{2}\right), \sigma_{d}\left(A_{1}\right)=\sigma_{d}\left(A_{2}\right), \sigma_{c}\left(A_{1}\right)=\sigma_{c}\left(A_{2}\right), \sigma_{r}\left(A_{1}\right)=\sigma_{r}\left(A_{2}\right)$, где $\sigma\left(A_{i}\right)$ - cпектр, $\sigma_{d}\left(A_{i}\right)$ - дискретный спектр, $\sigma_{c}\left(A_{i}\right)$-непрерывный спектр, $\sigma_{r}\left(A_{i}\right)$-остаточный спектр onepamopa $A_{i}, i=1,2$;

(2) если оператор $A_{2}$ допускает разложение $A_{2}=A_{21} \oplus A_{22}$, где $A_{2 k}=A \mid H_{k}, k=1,2$, - сужение $A_{2}$ на $H_{k}$ относительно прямой суммы $H=H_{1} \oplus H_{2}$ инвариантных относительно $A_{2}$ подпространств $H_{1}, H_{2}$, то подпространства $\tilde{H}_{k}=U\left(H_{k}\right), k=1,2$, инвариантны относительно оператора $A_{1}$ и $A_{1}=A_{11} \oplus A_{12}$, где $A_{1 k}=A_{1} \mid \tilde{H}_{k}, k=1,2 ;$ при этом $H=\tilde{H}_{1} \oplus \tilde{H}_{2}$. Кроме того, если $P$ - проектор, осуществляющий разложение $H=H_{1} \oplus H_{2}$ (m.е. $H_{1}=\operatorname{Im} P-$ образ проектора $P, H_{2}=\operatorname{Im}(I-P)$ - образ дополнительного проектора $\left.I-P\right)$, то проектор $\tilde{P} \in$ End $H$, осуществляющий разложение $H=\tilde{H}_{1} \oplus \tilde{H}_{2}$, определяется формулой $\tilde{P}=U P U^{-1}$.

Всюду в статье символом $A: D(A) \subset H \rightarrow H$ обозначается самосопряженный замкнутый оператор, в дальнейшем играющий роль невозмущенного оператора.

Определение 2.2. Оператор $B: D(B) \subset H \rightarrow H$ называется подчиненным оператору $A$, если $D(B) \supseteq D(A)$ и существует такая постоянная $C>0$, что $\|B x\| \leqslant C(\|x\|+\|A x\|)$ для любого $x \in D(A)$, где $C=C(A)$ - некоторая постоянная.

Множество линейных операторов, подчиненных оператору $A$, будем обозначать $L_{A}(H)$. Положим $\|B\|_{A}=\inf C$, где inf берется по всем постоянным $C>0$ из определения 2.2. Эта величина конечна и принимается за норму оператора $B$ в $L_{A}(H)$.

В работе используется термин трансформатор, который согласно терминологии М. Г. Крейна обозначает линейный оператор в пространстве линейных операторов. Область определения трансформатора состоит из таких операторов $X \in \operatorname{End} H$, для которых $X D(A) \subset D(A)$ и оператор $Y=A X-X A: D(A) \rightarrow H$ допускает ограниченное расширение $Y$ на $H$.

Основной составляющей метода подобных операторов является понятие допустимой тройки, которая должна для применимости метода удовлетворять ряду условий.

Определение 2.3. [3] Пусть $\mathfrak{A}$-линейное многообразие операторов из $L_{A}(H)$ и $J: \mathfrak{A} \rightarrow \mathfrak{A}$, $\Gamma: \mathfrak{A} \rightarrow$ End $H$ - линейные трансформаторы. Тройку $(\mathfrak{A}, J, \Gamma)$ назовем допустимой тройкой для оператора $A: D(A) \subset H \rightarrow H$, а $\mathfrak{A}$ - пространством допустимых возмущений, если выполнены следующие условия: 
(1) $\mathfrak{A}$ - банахово пространство с нормой $\|\cdot\|_{*}$, непрерывно вложенное в $L_{A}(H)$ (т.е. существует такая постоянная $C>0$, что $\|X\|_{A} \leqslant C\|X\|_{*}$, для любого $\left.X \in L_{A}(X)\right)$;

(2) $J$ и $\Gamma$ - непрерывные трансформаторы, причем $J$ - проектор;

(3) $(\Gamma X) D(A) \subset D(A)$ и, более того, имеют место равенства

$$
A \Gamma X-(\Gamma X) A=X-J X, \quad X \in \mathfrak{A},
$$

справедливые для векторов из $D(A)$, причем $\Gamma X$ - единственное решение уравнения $A Y-$ $Y A=X-J X$, удовлетворяющее условию $J Y=0$;

(4) $X \Gamma Y,(\Gamma X) Y \in \mathfrak{A}$ для любых $X, Y \in \mathfrak{A}$ и существует такая постоянная $\gamma>0$, что $\|\Gamma\| \leqslant \gamma$ и

$$
\max \left\{\|X \Gamma Y\|_{*},\|(\Gamma X) Y\|_{*}\right\} \leqslant \gamma\|X\|_{*}\|Y\|_{*} \quad \forall X, Y \in \mathfrak{A} ;
$$

(5) $J((\Gamma X) J Y)=0$ для всех $X, Y \in \mathfrak{A}$;

(6) для любых $X \in \mathfrak{A}$ и $\varepsilon>0$ можно указать такое число $\lambda_{\varepsilon} \in \rho(A)$, что $\left\|X\left(A-\lambda_{\varepsilon} I\right)^{-1}\right\|<\varepsilon$, где $I$ - тождественный оператор.

Теорема 2.1. Пусть (A, J, Г) - допустимая тройка для невозмущенного оператора

$$
A: D(A) \subset H \rightarrow H
$$

и $B \in \mathfrak{A}$. Если выполняется неравенство

$$
\|J\|\|B\|_{*}\|\Gamma\|<\frac{1}{4}
$$

то оператор вида $A-B$ подобен оператору вида $A-J \tilde{X}$, где $\tilde{X} \in \mathfrak{A}$ является решение нелинейного уравнения

$$
X=B \Gamma X-(\Gamma X) J(B \Gamma X)-(\Gamma X) J B+B=\Phi(X),
$$

которое можно найти методом простых итераиий, полагая $X_{0}=0, X_{1}=B, X_{2}=\Phi\left(X_{1}\right)$, $\ldots, X_{n+1}=\Phi\left(X_{n}\right), n \geqslant 2$. Отображение $\Phi: \mathfrak{A} \rightarrow \mathfrak{A}$ является сжимающим в шаре $\{X \in \mathfrak{A}:$ $\left.\|X-B\|_{*} \leqslant 3\|B\|_{*}\right\}$. Преобразование подобия оператора $A-B$ в оператор $A-J \tilde{X}$ осуществляет обратимый оператор $I+\Gamma \tilde{X}$, т.е. имеет место равенство

$$
(A-B)(I+\Gamma \tilde{X})=(I+\Gamma \tilde{X})(A-J \tilde{X}) .
$$

Далее в статье производится построение допустимой тройки метода подобных операторов и исследуются спектральные свойства возмущенного оператора.

3. Построение допустимой тройки метода подобных операторов для одного класса неограниченных возмущений. Для самосопряженного оператора $A: D(A) \subset H \rightarrow H$, имеющего спектр, состоящий из множеств $\sigma_{n}=\left\{\lambda_{n}\right\}$, где $\lambda_{n}$ - полупростые собственные значения удовлетворяющие условию (1), построим допустимую тройку ( $\mathfrak{A}, J, \Gamma)$.

Построение допустимой тройки осуществляется с привлечением ортогональных проекторов Рисса $P_{k}, k \geqslant 1$, построенных по спектральным множествам $\sigma_{n}=\left\{\lambda_{n}\right\}$, т.е. $A P_{k}=\lambda_{k} P_{k}, k \geqslant 1$. Проекторы Рисса $P_{k}, k \geqslant 1$, построенные по изолированным спектральным множествам $\sigma_{n}$, приводят оператор $A$ и разлагают пространство $H$ в ортогональную прямую сумму подпространств $H_{k}, H=H_{1} \oplus H_{2} \oplus \ldots$, где $H_{k}=\operatorname{Im} P_{k}$.

В случае $\operatorname{dim} \operatorname{Im} P_{k}=\operatorname{dim} H_{k}=2$ будем получать проекторы вида

$$
P_{k} x=\left(x, e_{k}\right) e_{k}+\left(x, e_{-k}\right) e_{-k}, \quad k \geqslant 1, \quad e_{k} \perp e_{-k}, \quad\left\|e_{k}\right\|=\left\|e_{-k}\right\|=1,
$$

где $A e_{k}=\lambda_{k} e_{k}, A e_{-k}=\lambda_{k} e_{-k}, k \geqslant 1$. Через $A_{k}=\lambda_{k} I_{k}$ будем обозначать сужение оператора $A$ на подпространство $H_{k}$.

Матрица оператора $A$ в базисе из собственных векторов в общем случае имеет блочно-диагональный вид.

Введем в рассмотрение такой проектор $P_{m}^{\prime}$, что $P_{m}^{\prime}=P_{1}+P_{2}+\cdots+P_{m}$ и $H_{m}^{\prime}=\operatorname{Im} P_{m}^{\prime}$, $\operatorname{dim} H_{m}^{\prime}=\sum_{i=1}^{m} \operatorname{dim} H_{i}$, т.е. $P_{m}^{\prime}$ - проектор Рисса, построенный по множеству $\sigma_{m}^{\prime}=\left\{\lambda_{1}, \lambda_{2}, \ldots, \lambda_{m}\right\}$. 
Значение $m$ выбирается с тем расчетом, чтобы $d_{m}=\operatorname{dist}\left(\sigma_{m}^{\prime}, \sigma(A) \backslash \sigma_{m}^{\prime}\right)$ было достаточно велико. Тогда спектр оператора $A$ представим в виде

$$
\sigma(A)=\sigma_{m}^{\prime} \cup\left(\bigcup_{n=m+1}^{\infty} \sigma_{n}\right) .
$$

Через $A_{n}=\lambda_{n} I_{n}$ будем обозначать сужение оператора $A$ на подпространство $H_{n}, n \geqslant m+1$, а через $A P_{m}^{\prime}$ - сужение на подпространство $H_{m}^{\prime}=\operatorname{Im} P_{m}^{\prime}$.

Построение рассматриваемого пространства допустимых возмущение $\mathfrak{A}$ осуществляется следующим образом. Оператор $X$ из $L_{A}(H)$ отнесем к $\mathfrak{A}$, если выполняются следующие условия:

(1) $\sup \left\|P_{m} X P_{k}\right\|=M(n)<\infty$ для всех $n \in \mathbb{Z}, m, k \geqslant 1$;

(2) $\sum_{n \in \mathbb{Z}}^{m} M^{2}(n)<\infty$.

В качестве нормы в $\mathfrak{A}$ берется величина

$$
\|X\|_{*}=\sqrt{\sum_{n \in \mathbb{Z}} M^{2}(n)} .
$$

Из условия (1) следует сильная сходимость ряда

$$
\sum_{m-k=n} P_{m} X P_{k}, \quad m, k \geqslant 1,
$$

к некоторому оператору $X(n)$ из End $H$. Этот оператор назовем $n$-й диагональю оператора $X$. Действительно, для любого $X \in \mathfrak{A}$ и $x \in H$ верны следующие неравенства, полученные при помощи равенства Парсеваля (для определенности будем считать, что $n \geqslant 0$ ):

$$
\begin{aligned}
\|X(n) x\|^{2}=\sum_{m-k=n}\left\|P_{m} X P_{k} x\right\|^{2}= & \sum_{m=n+k}^{\infty}\left\|P_{m} X P_{m-n} x\right\|^{2} \leqslant \\
& \leqslant \sup _{m \geqslant n+k}\left\|P_{m} X P_{m-n} x\right\|^{2}\|x\|^{2} \leqslant M^{2}(n)\|x\|^{2}, \quad m, k \geqslant 1 .
\end{aligned}
$$

Отсюда следует сходимость ряда (5) и неравенство $\|X(n)\| \leqslant M(n)$. Таким образом, каждый оператор $X \in \mathfrak{A}$ допускает представление вида

$$
X=\sum_{n=-\infty}^{\infty} X(n)
$$

и его блоки $X_{m k}=P_{m} X P_{k}$, стоящие на $n$-й диагонали $(m-k=n)$ равномерно ограничены. Сходимость ряда (6) понимается на векторах из $D(A)$, причем

$$
X x=\lim _{n \rightarrow \infty} \sum_{k=-n}^{n} X(k) x, \quad x \in D(A) .
$$

Факт $X \in \mathfrak{A}$ обоснован в следующей теореме.

Теорема 3.1. Пространство $\mathfrak{A}$ является банаховым и оно непрерывно вложено в $L_{A}(H)$.

Доказательство. Выполнение аксиом нормы для пространства $\mathfrak{A}$ проверить несложно; покажем, что оно полно относительно введенной нормы. Если $\left(X_{l}\right)$ - фундаментальная последовательность в $\mathfrak{A}$, то она ограничена в $\mathfrak{A}$ и поэтому величина

$$
\sup _{m-k=n}\left\|P_{m} X_{l} P_{k}\right\|=\left\|X_{l}(n)\right\|=M_{l}(n), \quad n \in \mathbb{Z}, \quad m, k \geqslant 1,
$$

будет обладать свойством

$$
\left\|X_{l}\right\|_{*}^{2}=\sup _{l \geqslant 1} \sum_{n=-\infty}^{\infty} M_{l}^{2}(n)<\infty .
$$


Из этой оценки следует, что последовательность операторов $\left(X_{l}(n)\right)$ будет фундаментальной в End $H$ для каждого фиксированного $n \in \mathbb{Z}$. Обозначим предел этой последовательности через $X_{0}(n)$; тогда

$$
\sum_{n=-\infty}^{\infty}\left\|X_{0}(n)\right\|^{2} \leqslant \sup _{l \geqslant 1}\left\|X_{l}\right\|_{*}^{2}<\infty
$$

На векторах $x \in D(A)$ определим оператор $X_{0}: D(A) \rightarrow H$ формулой

$$
X_{0} x=\sum_{n=-\infty}^{\infty} X_{0}(n) x .
$$

Докажем, что $X_{0} \in L_{A}(H)$. Пусть $\lambda_{0} \in \rho(A)$. Тогда верны равенства

$$
X_{0}(n) x=X_{0}(n)\left(A-\lambda_{0} I\right)^{-1}\left(A-\lambda_{0} I\right) x=\left(\sum_{\substack{m-k=n, m, k \geqslant 1}} \frac{P_{m} X_{0} P_{k}}{\lambda_{k}-\lambda_{0}}\right) y,
$$

где $y=\left(A-\lambda_{0} I\right) x$. Так как последовательность $\left\{\lambda_{k}\right\}$ удовлетворяет соотношению (1), то последовательность $\left(\alpha_{k}\right)$, где $\alpha_{k}=1 /\left|\lambda_{k}-\lambda_{0}\right|, k>0$, принадлежит $l_{2}$.

Определим оператор $Y_{0}$ формулой

$$
Y_{0}=\sum_{n=-\infty}^{\infty} Y_{0}(n)=\sum_{n=-\infty}^{\infty} \sum_{m-k=n} \frac{P_{m} X_{0} P_{k}}{\lambda_{k}-\lambda_{0}}, \quad m, k \geqslant 1,
$$

где $X_{0} \in \mathfrak{A}$. Факт $Y_{0} \in$ End $H$ следует из соотношения

$$
Y_{0}(n)=\sum_{m-k=n} \frac{P_{m} X_{0} P_{k}}{\lambda_{k}-\lambda_{0}}, \quad m, k \geqslant 1,
$$

из которого получаем:

$$
\left\|Y_{0}(n)\right\|=\sup _{m-k=n}\left\|P_{m} Y_{0} P_{k}\right\| \leqslant \sup _{m-k=n}\left\|P_{m} X_{0} P_{k}\right\|\left\|\left(\alpha_{k}\right)\right\|_{l_{2}}=\tilde{M}(n)<\infty, \quad m, k \geqslant 1 .
$$

Следовательно,

$$
\left\|Y_{0}\right\|^{2} \leqslant \sum_{n=-\infty}^{\infty}\left\|Y_{0}(n)\right\|^{2}=\sum_{n=-\infty}^{\infty} \tilde{M}^{2}(n)<\infty .
$$

Поскольку $X_{0}=Y_{0}\left(A-\lambda_{0} I\right)$, т.е. $\left\|X_{0} x\right\| \leqslant C(\|A x\|+\|x\|), x \in D(A)$, то $X_{0} \in L_{A}(H)$. Получаем из доказанного, что $\mathfrak{A}$ непрерывно вложено в $L_{A}(H)$ и справедлива оценка

$$
\left\|X_{0}\right\|_{*} \leqslant C\left\|X_{0}\left(A-\lambda_{0} I\right)^{-1}\right\|_{\text {End } H}, \quad X_{0} \in \mathfrak{A} .
$$

Построение трансформатора $J: \mathfrak{A} \rightarrow \mathfrak{A}$ также будет зависеть от выбранного $m$, поэтому обозначим его $J_{m}$ и на операторах из допустимого пространства возмущений определим формулой

$$
J_{m} X=P_{m}^{\prime} X P_{m}^{\prime}+\sum_{i=m+1}^{\infty} P_{i} X P_{i}, \quad X \in \mathfrak{A} ;
$$

$J$ является проектором. Соответственно трансформатор $\Gamma=\Gamma_{m}: \mathfrak{A} \rightarrow$ End $H$ в этом случае определяется формулой

$$
\Gamma_{m} X=\sum_{i=m+1}^{\infty} \sum_{j=1}^{i-1} \frac{P_{i} X P_{j}}{\lambda_{i}-\lambda_{j}}+\sum_{j=m+1}^{\infty} \sum_{i=1}^{j-1} \frac{P_{i} X P_{j}}{\lambda_{i}-\lambda_{j}} .
$$

Найдем представление для $\Gamma_{m} X(n)$ при $n \neq 0$ : 


$$
\begin{gathered}
\Gamma_{m} X(n)=\sum_{i=m+1}^{\infty} \sum_{j=1}^{i-1} \frac{P_{i}\left(\sum_{l-k=n} P_{l} X P_{k}\right) P_{j}}{\lambda_{i}-\lambda_{j}}+\sum_{j=m+1}^{\infty} \sum_{i=1}^{j-1} \frac{P_{i}\left(\sum_{l-k=n} P_{l} X P_{k}\right) P_{j}}{\lambda_{i}-\lambda_{j}}= \\
=\sum_{i=m+1}^{\infty} \sum_{\substack{j=1, i-j=n}}^{i-1} \frac{P_{i} X P_{j}}{\lambda_{i}-\lambda_{j}}+\sum_{j=m+1}^{\infty} \sum_{\substack{i=1, i-j=n}}^{j-1} \frac{P_{i} X P_{j}}{\lambda_{i}-\lambda_{j}} .
\end{gathered}
$$

Оценим норму трансформатора $\Gamma=\Gamma_{m}: \mathfrak{A} \rightarrow$ End $H$ при каждом $m \in \mathbb{N}$. Из представления оператора $\Gamma_{m} X(n)$ с учетом того факта, что это $n$-диагональный ограниченный оператор, получаем оценку вида

$$
\left\|\Gamma_{m} X(n)\right\|=\alpha_{m}(n) \sup _{i-j=n}\left\|P_{i} X P_{j}\right\| \leqslant \alpha_{m}(n)\|X(n)\|,
$$

где двусторонняя последовательность $\alpha_{m}: \mathbb{Z} \rightarrow \mathbb{R}$ определяется равенством

$$
\alpha_{m}(n)=\max \left(\max _{\substack{i>m, 1 \leqslant j \leqslant i-1}}\left(\left|\lambda_{i}-\lambda_{j}\right|^{-1}\right) ; \max _{\substack{j>m, 1 \leqslant i \leqslant j-1}}\left(\left|\lambda_{i}-\lambda_{j}\right|^{-1}\right)\right), \quad i-j=n, \quad n \neq 0 .
$$

В дальнейшем всюду считаются выполненными следующие условия:

(1) двусторонняя последовательность $\alpha_{m}$ принадлежит $l_{2}(\mathbb{Z})$;

(2) $\lim _{m \rightarrow \infty}\left\|\alpha_{m}\right\|_{l_{2}}=0$.

Из этих условий следует, что спектр рассматриваемого оператора удовлетворяет условию (1).

Таким образом, получаем

$$
\begin{aligned}
\left\|\Gamma_{m} X\right\|=\sum_{n=-\infty}^{\infty}\left\|\Gamma_{m} X(n)\right\| \leqslant \sum_{n=-\infty}^{\infty} \alpha_{m}(n)\|X(n)\| \leqslant \\
\leqslant \sqrt{\sum_{n=-\infty}^{\infty} \alpha_{m}^{2}(n)} \sqrt{\sum_{n=-\infty}^{\infty}\|X(n)\|^{2}} \leqslant \sqrt{\sum_{n=-\infty}^{\infty} \alpha_{m}^{2}(n)\|X\|_{*}}=\left\|\alpha_{m}\right\|_{l_{2}(\mathbb{Z})}\|X\|_{*} .
\end{aligned}
$$

Следовательно, трансформатор $\Gamma_{m}$ допускает оценку

$$
\left\|\Gamma_{m}\right\| \leqslant \sqrt{\sum_{n=-\infty}^{\infty} \alpha_{m}^{2}(n)}=\left\|\alpha_{m}\right\|_{l_{2}(\mathbb{Z})}
$$

Из этой оценки следует, что $\lim _{m \rightarrow \infty}\left\|\Gamma_{m}\right\|=0$. Тогда, выбирая достаточно большое $m$, можно не требовать малости $\|B\|_{*}$.

Лемма 3.1. Тройка $\left(\mathfrak{A}, J_{m}, \Gamma_{m}\right)$ является допустимой для оператора $A: D(A) \subset H \rightarrow H u$

$$
\begin{gathered}
\left\|\Gamma_{m}\right\| \leqslant\left\|\alpha_{m}\right\|_{l_{2}(\mathbb{Z})} \\
\max \left\{\left\|X \Gamma_{m} Y\right\|_{*},\left\|\left(\Gamma_{m} X\right) Y\right\|_{*}\right\} \leqslant\left\|\alpha_{m}\right\|_{l_{2}(\mathbb{Z})}\|X\|_{*}\|Y\|_{*}, \quad X, Y \in \mathfrak{A} .
\end{gathered}
$$

Доказательство. Первое свойство допустимой тройки, т.е. факт, что банахово пространство $\mathfrak{A}$ непрерывно вложено в $L_{A}(H)$, доказано в теореме 3.1. Из построения $J_{m}$ и $\Gamma_{m}$ видно, что транс-

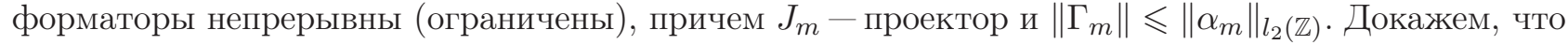
$\left(\Gamma_{m} X\right) D(A) \subset D(A)$ и $A \Gamma_{m} X-\Gamma_{m} X A=X-J_{m} X$ для всех $X \in \mathfrak{A}$. В соответствии с построением пространства возмущений $\mathfrak{A}$, если $X \in \mathfrak{A}$, то диагонали $X(n), n \geqslant 1$, этого оператора равномерно ограничены, откуда следует ограниченность $\Gamma_{m} X(n)$. Следовательно, $\left(\Gamma_{m} X(n)\right) D(A) \subset D(A)$ (см. [1], где доказано это свойство для ограниченных операторов).

Рассмотрим последовательность операторов $\tilde{X}_{n}=\sum_{k=-n}^{n} X(k), n \geqslant 0 ;$ имеем

$$
X x=\lim _{n \rightarrow \infty} \tilde{X}_{n}, \quad x \in D(A),
$$


где $X \in \mathfrak{A}$.

Пусть $x \in D(A)$; как в теореме 3.1 , рассмотрим $\lambda_{0} \in \rho(A)$. Тогда

$$
\Gamma_{m} \tilde{X}_{n} x=\Gamma_{m} \tilde{X}_{n}\left(A-\lambda_{0} I\right)^{-1}\left(A-\lambda_{0} I\right) x=\Gamma_{m} \tilde{X}_{n}\left(A-\lambda_{0} I\right)^{-1} y,
$$

где $y \in H$.

Рассмотрим последовательность векторов вида $z_{n}=\Gamma_{m} \tilde{X}_{n}\left(A-\lambda_{0} I\right)^{-1} y, z_{n} \in D(A)$. Поскольку

$$
\lim _{n \rightarrow \infty} \tilde{X}_{n}\left(A-\lambda_{0} I\right)^{-1} y=X\left(A-\lambda_{0} I\right)^{-1} y,
$$

то $z_{n} \rightarrow z=\Gamma_{m} X\left(A-\lambda_{0} I\right)^{-1} y$. Таким образом, последовательность векторов $g_{n}=A z_{n}, n \geqslant 1$, такова, что $g_{n} \rightarrow g_{0}=A z_{0}$. Так как $A$-замкнутый оператор, то из условий $z_{n} \rightarrow z_{0}$ и $A z_{n} \rightarrow g_{0}$ следует, что $z_{0} \in D(A)$. Итак, получили включение $\Gamma_{m} D(A) \subset D(A)$.

Докажем равенство

$$
A \Gamma_{m} X-\Gamma_{m} X A=X-J_{m} X, \quad X \in \mathfrak{A} .
$$

Введем обозначения $y=\left(A\left(\Gamma_{m} X\right)-\left(\Gamma_{m} X\right) A\right) x$ и $z=\left(X-J_{m} X\right) x, x \in D(A)$. Для доказательства (7) достаточно установить, что $P_{m}^{\prime} y=P_{m}^{\prime} z$. Используя перестановочность оператора $A$ и проекторов $P_{n}, n \geqslant 1$, имеем

$$
\begin{gathered}
P_{m}^{\prime} y=P_{m}^{\prime}\left(A\left(\Gamma_{m} X\right)-\left(\Gamma_{m} X\right) A\right) x=P_{m}^{\prime}\left(\sum_{i=m+1}^{\infty} \sum_{j=1}^{i-1} \frac{A P_{i} X P_{j}}{\lambda_{i}-\lambda_{j}}+\sum_{j=m+1}^{\infty} \sum_{i=1}^{j-1} \frac{A P_{i} X P_{j}}{\lambda_{i}-\lambda_{j}}\right) x- \\
-\left(\sum_{i=m+1}^{\infty} \sum_{j=1}^{i-1} \frac{P_{m}^{\prime} P_{i} X P_{j}}{\lambda_{i}-\lambda_{j}}+\sum_{j=m+1}^{\infty} \sum_{i=1}^{j-1} \frac{P_{m}^{\prime} P_{i} X P_{j}}{\lambda_{i}-\lambda_{j}}\right) A x= \\
=\sum_{j=m+1}^{\infty} \sum_{i=1}^{m} \frac{\lambda_{i} P_{i} X P_{j}}{\lambda_{i}-\lambda_{j}} x-\sum_{j=m+1}^{\infty} \sum_{i=1}^{m} \frac{\lambda_{j} P_{i} X P_{j}}{\lambda_{i}-\lambda_{j}} x=\sum_{j=m+1}^{\infty} \sum_{i=1}^{m} P_{i} X P_{j} x .
\end{gathered}
$$

Применяя проектор $P_{m}^{\prime}$ к вектору $z$, получаем

$$
\begin{aligned}
P_{m}^{\prime} z=P_{m}^{\prime}\left(X-J_{m} X\right) x=P_{m}^{\prime} X x-P_{m}^{\prime}\left(P_{m}^{\prime} X P_{m}^{\prime}+\sum_{i=m+1}^{\infty} P_{i} X P_{i}\right)= \\
=\sum_{i=1}^{m} P_{i} X \sum_{j=1}^{\infty} P_{j} x-\sum_{i, j=1}^{m} P_{i} X P_{j} x=\sum_{j=m+1}^{\infty} \sum_{i=1}^{m} P_{i} X P_{j} x .
\end{aligned}
$$

Пусть теперь $n>m$, тогда

$$
\begin{aligned}
& P_{n} y=P_{n}(\left.A\left(\Gamma_{m} X\right)-\left(\Gamma_{m} X\right) A\right) x=\left(\sum_{j=1}^{n-1} \frac{\lambda_{n} P_{n} X P_{j}}{\lambda_{n}-\lambda_{j}} x+\sum_{j=m+1}^{\infty} \frac{\lambda_{n} P_{n} X P_{j}}{\lambda_{n}-\lambda_{j}} x\right)- \\
&-\left(\sum_{j=1}^{n-1} \frac{\lambda_{j} P_{n} X P_{j}}{\lambda_{n}-\lambda_{j}} x+\sum_{j=m+1}^{\infty} \frac{\lambda_{j} P_{n} X P_{j}}{\lambda_{n}-\lambda_{j}} x\right)=\sum_{j=1}^{n-1} P_{n} X P_{j} x+\sum_{j=m+1}^{\infty} P_{n} X P_{j} x= \\
&=\sum_{j=1}^{n-1} P_{n} X P_{j} x-P_{n} X P_{n} x .
\end{aligned}
$$

Применим проектор $P_{n}, n>m$ к вектору $z$ :

$$
\begin{aligned}
P_{n} z=P_{n}\left(X-J_{m} X\right) x=P_{n} X x-P_{n}( & \left.P_{m}^{\prime} X P_{m}^{\prime}+\sum_{i=m+1}^{\infty} P_{i} X P_{i}\right) x= \\
& =P_{n} \sum_{i, j=1}^{\infty} P_{i} X P_{j} x-P_{n} X P_{n} x=\sum_{j=1}^{\infty} P_{n} X P_{j} x-P_{n} X P_{n} x .
\end{aligned}
$$


Получили $P_{n} y=P_{n} z, n \geqslant 1$; значит, равенство (7) выполнено.

Выполнение четвертого свойства допустимой тройки можно доказать, рассматривая оператор $Z=\left(\Gamma_{m} X\right) Y, X, Y \in \mathfrak{A}$, допускающий представление

$$
Z=\sum_{n=-\infty}^{\text {infty }} Z(n), \quad \text { где } Z(n)=\sum_{k \in \mathbb{Z}}\left(\Gamma_{m} X\right)(k) Y(n-k) .
$$

Приходим к оценкам

$$
\begin{gathered}
\|Z(n)\| \leqslant \sum_{k=-\infty}^{\infty}\left\|\left(\Gamma_{m} X\right)(k)\right\|\|Y(n-k)\| \leqslant \sum_{k=-\infty}^{\infty} \alpha_{m}(k)\|X(k)\|\|Y(n-k)\|, \\
\left\|\left(\Gamma_{m} X\right) Y\right\|_{*} \leqslant\left(\sum_{k=-\infty}^{\infty} \alpha_{m}^{2}(n)\right)^{1 / 2}\|X\|_{*}\|Y\|_{*},
\end{gathered}
$$

из которых следует, что $Z=\left(\Gamma_{m} X\right) Y \in \mathfrak{A} ;$ аналогично для $X \Gamma_{m} Y$. Константа $\gamma$ выбирается равной $\left\|\alpha_{m}\right\|_{l_{2}(\mathbb{Z})}$. Итак,

$$
\begin{gathered}
\left\|\Gamma_{m}\right\| \leqslant\left\|\alpha_{m}\right\|_{l_{2}(\mathbb{Z})} \\
\max \left\{\left\|X \Gamma_{m} Y\right\|_{*},\left\|\left(\Gamma_{m} X\right) Y\right\|_{*}\right\} \leqslant\left\|\alpha_{m}\right\|_{l_{2}(\mathbb{Z})}\|X\|_{*}\|Y\|_{*}, \quad X, Y \in \mathfrak{A} .
\end{gathered}
$$

Итак, тройка $\left(\mathfrak{A}, \Gamma_{m}, J_{m}\right)$ является допустимой для оператора $A$.

Из леммы 3.1 и теоремы 2.1 метода подобных операторов вытекает следующая теорема.

Теорема 3.2. Пусть $A: D(A) \subset H \rightarrow H-$ самосопряженный оператор со спектром, удовлетворяющим условию (1), оператор $B: D(B) \subset H \rightarrow H$ принадлежит построенному пространству допустимых возмущений $\mathfrak{A}$ и существует такое натуральное число $m_{0}$, что

$$
\left\|\alpha_{m}\right\|_{l_{2}(\mathbb{Z})}\|B\|_{*} \leqslant \frac{1}{4}
$$

Тогда оператор $A-B$ подобен оператору $A-J_{m_{0}} X_{0}$ и имеет место равенство

$$
(A-B)\left(I+\Gamma_{m_{0}} X_{0}\right)=\left(I+\Gamma_{m_{0}} X_{0}\right)\left(A-J_{m_{0}} X_{0}\right)=\left(I+\Gamma_{m_{0}} X_{0}\right)\left(A-P_{m_{0}}^{\prime} X P_{m_{0}}^{\prime}-\sum_{i \geqslant m_{0}+1}^{\infty} P_{i} X_{0} P_{i}\right),
$$

где $X_{0}$ - решение нелинейного уравнения вида (3), которое можно найти методом простых итераций.

В следующей теореме описан спектр возмущенного оператора $A-B$.

Теорема 3.3. Пусть $A: D(A) \subset H \rightarrow H-$ самосопряжсенный оператор со спектром $\sigma(A)=$ $\left\{\lambda_{1}, \lambda_{2}, \ldots\right\}, \lambda_{1}<\lambda_{2}<\ldots$, удовлетворяющим условию (1), и число т 0 таково, что выполнено условие (8). Спектр оператора А представим в виде

$$
\sigma(A)=\sigma_{m_{0}}^{\prime} \cup\left(\bigcup_{n=m_{0}+1}^{\infty} \sigma_{n}\right)
$$

где $\sigma_{m_{0}}^{\prime}=\left\{\lambda_{1}, \lambda_{2}, \ldots, \lambda_{m_{0}}\right\}, \sigma_{n}=\lambda_{n}, n \geqslant m_{0}+1$ ( $\lambda_{n}-$ полупростые собственные значения оператора $A)$. Тогда спектр оператора $A-B$ представим в виде обвединения

$$
\sigma(A-B)=\tilde{\sigma}_{m_{0}}^{\prime} \cup\left(\bigcup_{n=m_{0}+1}^{\infty} \tilde{\sigma}_{n}\right)
$$

взаимно не пересекаюшихся множеств $\widetilde{\sigma}_{m_{0}}^{\prime}, \widetilde{\sigma}_{n}^{\prime}, n \geqslant m_{0}+1$, обладающих свойством

$$
\begin{aligned}
& \operatorname{dist}\left(\widetilde{\sigma}_{m_{0}}^{\prime}, \sigma_{m_{0}}^{\prime}\right) \leqslant 4\|B\|_{*}, \\
& \operatorname{dist}\left(\widetilde{\sigma}_{n}, \widetilde{\sigma}_{n}^{0}\right) \leqslant 2\left\|P_{n} B\right\|_{*}\|B\|_{*}\left\|\alpha_{n-1}\right\|_{l_{2}(\mathbb{Z})}, \quad n \geqslant m_{0}+1,
\end{aligned}
$$

где $\widetilde{\sigma}_{n}^{0}=\sigma\left(\left.\left(\lambda_{n} P_{n}-P_{n} B\right)\right|_{H_{n}}\right)$. 
Доказательство. Поскольку

$$
H=H_{m_{0}}^{\prime} \oplus\left(\bigoplus_{n=m_{0}+1}^{\infty} H_{n}\right)
$$

спектр $\sigma\left(A-J_{m_{0}} X_{0}\right)$ оператора $A-J_{m_{0}} X_{0}$ содержит спектр оператора $\left.\left(A P_{m_{0}}^{\prime}-P_{m_{0}}^{\prime} X_{0}\right)\right|_{H_{m_{0}}^{\prime}}$ и каждого из операторов $\left.\left(A_{n}-P_{n} X_{0}\right)\right|_{H_{n}}$, действующих в $H_{n}, n \geqslant m_{0}+1$. Поскольку выполнены условия теоремы 3.2 , то оператор $A-B$ подобен оператору вида $A-J_{m_{0}} X_{0}$, где $X_{0}$ - решение нелинейного уравнения (3). Воспользовавшись равенством (9), где

$$
A-J_{m_{0}} X_{0}=A-P_{m_{0}}^{\prime} X_{0} P_{m_{0}}^{\prime}-\sum_{i=m_{0}+1}^{\infty} P_{i} X P_{i},
$$

получаем

$$
\begin{aligned}
& \sigma(A-B)=\sigma\left(A-J_{m_{0}} X_{0}\right)= \\
& \quad=\sigma\left(\left.\left(A P_{m_{0}}^{\prime}-P_{m_{0}}^{\prime} X_{0}\right)\right|_{H_{m_{0}}^{\prime}}\right) \cup\left(\bigcup_{n \geqslant m_{0}+1} \sigma\left(\left.\left(\lambda_{n} I_{n}-P_{n} X_{0}\right)\right|_{H_{n}}\right)\right)=\widetilde{\sigma}_{m_{0}}^{\prime} \cup\left(\bigcup_{n=m_{0}+1}^{\infty} \tilde{\sigma}_{n}\right)
\end{aligned}
$$

для всех $m_{0}$, удовлетворяющих оценке (8), где

$$
\widetilde{\sigma}_{m_{0}}^{\prime}=\sigma\left(\left.\left(A P_{m_{0}}^{\prime}-P_{m_{0}}^{\prime} X_{0}\right)\right|_{H_{m_{0}}^{\prime}}\right), \quad \widetilde{\sigma}_{n}=\sigma\left(\left.\left(\lambda_{n} I_{n}-P_{n} X_{0}\right)\right|_{H_{n}}\right) .
$$

Эти множества взаимно не пересекаются.

Теперь получим оценку (11). Учитывая оценки в теореме 2.1, заключаем, что имеет место оценка

$$
\operatorname{dist}\left(\widetilde{\sigma}_{m_{0}}^{\prime}, \sigma_{m_{0}}^{\prime}\right) \leqslant\left\|P_{m_{0}}^{\prime} X_{0} P_{m_{0}}^{\prime}\right\| \leqslant\left\|X_{0}\right\| \leqslant 4\|B\|_{*} .
$$

Для получения оценок множеств $\widetilde{\sigma}_{n}, n \geqslant m_{0}+1$, для каждого $n>m_{0}$ будем рассматривать свою допустимую тройку $\left(\mathfrak{A}, J_{n}, \Gamma_{n}\right)$; поскольку $n \geqslant m_{0}+1$, то имеет место утверждение теоремы $3.2 \mathrm{c}$ последующими равенствами (13), где вместо $m_{0}$ берется $n$. Операторы $\lambda_{n} P_{n}-P_{n} X_{0} P_{n}, n \geqslant m_{0}+1$, представим в виде

$$
\lambda_{n} P_{n}-P_{n} X_{0} P_{n}=\lambda_{n} P_{n}-P_{n} B P_{n}-P_{n}\left(X_{0}-B\right) P_{n}, \quad n>m_{0} .
$$

Такое представление позволяет получить оценки

$$
\operatorname{dist}\left(\widetilde{\sigma}_{n}, \sigma\left(\left.\left(\lambda_{n} I_{n}-P_{n} X_{0}\right)\right|_{H_{n}}\right)\right) \leqslant\left\|P_{n}\left(X_{0}-B\right) P_{n}\right\|, \quad n \geqslant m_{0}+1 .
$$

Далее, имеют место равенства, вытекающие после применения слева и справа в (3) операторов $P_{n}, n \geqslant m_{0}+1:$

$$
P_{n}\left(X_{0}-B\right) P_{n}=P_{n} B \Gamma_{n-1}\left(X_{0} P_{n}\right), \quad n \geqslant m_{0}+1 .
$$

При этом учитывались равенства $P_{n}\left(\Gamma_{n-1} X_{0}\right)\left(J_{n-1} X_{0}\right) P_{n}=0, n \geqslant m_{0}+1$. Итак, получаем следующую оценку:

$$
\left\|P_{n}\left(X_{0}-B\right) P_{n}\right\| \leqslant\left\|P_{n} B \Gamma_{n-1}\left(X_{0} P_{n}\right)\right\| \leqslant\left\|P_{n} B\right\|\left\|\Gamma_{n-1}\left(X_{0} P_{n}\right)\right\|, \quad n \geqslant m_{0}+1 .
$$

Представим операторы $\Gamma_{n-1}\left(X_{0} P_{n}\right), n \geqslant m_{0}+1$ в виде

$$
\Gamma_{n-1}\left(X_{0} P_{n}\right)=\sum_{\substack{i=1, i \neq n}}^{\infty} \frac{P_{i} X_{0} P_{n}}{\lambda_{i}-\lambda_{n}} .
$$

Тогда получаем оценки 


$$
\begin{aligned}
& \left\|\Gamma_{n-1}\left(X_{0} P_{n}\right)\right\| \leqslant \sum_{\substack{i=1, i \neq n}}^{\infty} \frac{\left\|P_{i} X_{0} P_{n}\right\|}{\left|\lambda_{i}-\lambda_{n}\right|} \leqslant \sum_{\substack{i=1, i \neq n}}^{\infty} \frac{\left\|X_{0}(i-n)\right\|}{\lambda_{i}-\lambda_{n}} \leqslant \\
& \leqslant \sqrt{\sum_{i=1}^{\infty}\left\|X_{0}(k)\right\|^{2}} \sqrt{\sum_{\substack{i=1, i \neq n}}^{\infty} \frac{1}{\left|\lambda_{i}-\lambda_{n}\right|^{2}}} \leqslant \frac{1}{2}\left\|X_{0}\right\|_{*}\left\|\alpha_{n-1}\right\|_{l_{2}(\mathbb{Z}), \quad n \geqslant m_{0}+1 .}
\end{aligned}
$$

Вводя обозначение $\widetilde{\sigma}_{n}^{0}=\sigma\left(\left.\left(\lambda_{n} P_{n}-P_{n} B\right)\right|_{H_{n}}\right)$ из равенства (11) получаем оценку

$$
\operatorname{dist}\left(\widetilde{\sigma}_{n}, \widetilde{\sigma}_{n}^{0}\right) \leqslant\left\|P_{n} B \Gamma_{n-1}\left(X_{0} P_{n}\right)\right\| \leqslant\left\|P_{n} B\right\|_{*}\left\|\Gamma_{n-1}\left(X_{0} P_{n}\right)\right\| \leqslant \frac{1}{2}\left\|P_{n} B\right\|_{*}\left\|X_{0}\right\|_{*}\left\|\alpha_{n-1}\right\|_{l_{2}(\mathbb{Z})} .
$$

Учитывая полученные соотношения, а также (8), получаем оценки

$$
\operatorname{dist}\left(\widetilde{\sigma}_{n}, \widetilde{\sigma}_{n}^{0}\right) \leqslant 2\left\|P_{n} B\right\|_{*}\|B\|_{*}\left\|\alpha_{n-1}\right\|_{l_{2}(\mathbb{Z})}, \quad n \geqslant m_{0}+1 .
$$

4. Применение к дифференциальным операторам с периодическими краевыми условиями. Будем рассматривать в гильбертовом пространстве $L_{2}(0,2 \pi)$ суммируемых с квадратом функций со скалярным произведением

$$
(x, y)=\int_{0}^{2 \pi} x(t) \bar{y}(t) d t
$$

дифференциальный оператор

$$
A-B: D(A) \subset L_{2}(0,2 \pi) \rightarrow L_{2}(0,2 \pi), \quad A-B=-\frac{d^{2}}{d t^{2}}-q
$$

в случае периодических краевых условий

$$
x(0)=x(2 \pi), \quad x^{\prime}(0)=x^{\prime}(2 \pi) .
$$

Для изучения оператора будем использовать полученные в предыдущем разделе результаты, взяв в качестве невозмущенного самосопряженный оператор

$$
A: D(A) \subset L_{2}(0,2 \pi) \rightarrow L_{2}(0,2 \pi), \quad A=-\frac{d^{2}}{d t^{2}} .
$$

Область определения $D(A)$ оператора $A$ совпадает с пространством Соболева $W_{2}^{2}[0,2 \pi]$ непрерывно дифференцируемых функций $x:[0,2 \pi] \rightarrow \mathbb{C}$, производная которых $x^{\prime}$ абсолютно непрерывна и $x^{\prime \prime} \in L_{2}(0,2 \pi)$. Таким образом,

$$
D(A)=\left\{x: x \in W_{2}^{2}[0,2 \pi], x(0)=x(2 \pi), x^{\prime}(0)=x^{\prime}(2 \pi)\right\} .
$$

Оператор $B: D(A) \subset L_{2}(0,2 \pi) \rightarrow L_{2}(0,2 \pi)$ является оператором умножения на функцию $q$, принадлежащую пространству $L_{2}(0,2 \pi) \backslash L_{\infty}(0,2 \pi)$, т.е. $B$ не допускает ограниченное расширение на $L_{2}(0,2 \pi)$. Итак, $B$ - неограниченный оператор. Известно, что спектр оператора $A$ имеет вид

$$
\sigma(A)=\bigcup_{n=0}^{\infty} \sigma_{n},
$$

где $\sigma_{n}=\left\{n^{2}\right\}, n \geqslant 0$. При этом собственное значение $\lambda_{0}=0$ является однократным собственным значением, а $\lambda_{n}=n^{2}, n \geqslant 1,-$ собственными значениями кратности 2 .

Ортонормированные собственные функции $e_{n}(t)=(\sqrt{2 \pi})^{-1} e^{i n t}, n \in \mathbb{Z}, t \in[0,2 \pi]$, образуют ортонормированный базис в гильбертовом пространстве $L_{2}(0,2 \pi)$, причем $A e_{0}=0, A e_{n}=n^{2} e_{n}$, $|n| \geqslant 1$. Проекторы Рисса $P_{n}, n \geqslant 0$, построенные по этому базису, определяются равенствами

$$
P_{0} x=\left(x, e_{0}\right) e_{0}, \quad P_{n} x=\left(x, e_{n}\right) e_{n}+\left(x, e_{-n}\right) e_{-n}, \quad n \geqslant 1, \quad x \in L_{2}(0,2 \pi) .
$$


Оператор $B$ будет принадлежать построенному в разделе 3 пространству возмущений $\mathfrak{A}$, если будут выполнены соотношения

$$
\sup _{\substack{m-k=n, m, k \geqslant 1}}\left\|P_{m} B P_{k}\right\|=M(n)<\infty, \quad \sum_{n=-\infty}^{\infty} M^{2}(n)<\infty .
$$

Проверим выполнение этих свойств. Операторы $P_{m} B P_{k}, m, k \geqslant 1$, имеют вид

$$
\begin{aligned}
& P_{m} B P_{k} x=\left(B P_{k} x, e_{m}\right) e_{m}+\left(B P_{k} x, e_{-m}\right) e_{-m}= \\
& \quad=\left(\left(x, e_{k}\right)\left(B e_{k}, e_{m}\right)+\left(x, e_{-k}\right)\left(B e_{-k}, e_{m}\right)\right) e_{m}+\left(\left(x, e_{k}\right)\left(B e_{k}, e_{-m}\right)+\left(x, e_{-k}\right)\left(B e_{-k}, e_{-m}\right)\right) e_{-m},
\end{aligned}
$$

где

$$
\left(B e_{k}, e_{m}\right)=\left(q e_{k}, e_{m}\right)=\frac{1}{2 \pi} \int_{0}^{2 \pi} q(t) e^{-i(m-k) t} d t=\frac{1}{\sqrt{2 \pi}}\left(q, e_{m-k}\right), \quad\left(q, e_{m-k}\right)=\hat{q}(m-k)
$$

- коэффициенты Фурье функции $q$ по ортонормированному базису $\left(e_{n}\right)$. Таким образом, операторы $P_{m} B P_{k}, m, n \geqslant 1$, допускают представление вида

$$
\begin{aligned}
P_{m} B P_{k} x=\frac{1}{\sqrt{2 \pi}}\left(\left(x, e_{k}\right) \hat{q}(m-k)+\left(x, e_{-k}\right) \hat{q}(m+k)\right) e_{m}+ & \\
& +\frac{1}{\sqrt{2 \pi}}\left(\left(x, e_{k}\right) \hat{q}(-(m+k))+\left(x, e_{-k}\right) \hat{q}(-(m-k))\right) e_{-m} .
\end{aligned}
$$

Представления для блоков вида $P_{0} B P_{0}, P_{0} B P_{m}, P_{m} B P_{0}$ имеют вид

$$
\begin{aligned}
P_{0} B P_{0} x & =\frac{1}{\sqrt{2 \pi}}\left(x, e_{0}\right) \hat{q}(0) e_{0}, \\
P_{0} B P_{m} x & =\frac{1}{\sqrt{2 \pi}}\left(\left(x, e_{m}\right) \hat{q}(-m)+\left(x, e_{-m}\right) \hat{q}(m)\right) e_{0}, \\
P_{m} B P_{0} x & =\frac{1}{\sqrt{2 \pi}}\left(x, e_{0}\right)\left(\hat{q}(m) e_{m}+\hat{q}(-m) e_{-m}\right) .
\end{aligned}
$$

Отсюда вытекает оценка

$$
\left\|P_{m} B P_{k} x\right\|^{2}=\frac{1}{2 \pi}\left(|\hat{q}(m-k)|^{2}+|\hat{q}(m+k)|^{2}+|\hat{q}(-(m+k))|^{2}+|\hat{q}(-(m-k))|^{2}\right)\|x\|^{2} .
$$

Следовательно, имеют место оценки

$$
\left\|P_{m} B P_{k}\right\| \leqslant \frac{1}{\sqrt{2 \pi}}\left(|\hat{q}(m-k)|^{2}+|\hat{q}(m+k)|^{2}+|\hat{q}(-(m+k))|^{2}+|\hat{q}(-(m-k))|^{2}\right)^{1 / 2} .
$$

Так как $q \in L_{2}(0,2 \pi)$, то

$$
\|q\|=\left(\sum_{n=-\infty}^{\infty}|\hat{q}(n)|^{2}\right)^{1 / 2}
$$

Следовательно,

$$
\begin{aligned}
\|B\|_{*}=\left(\sum_{n=-\infty}^{\infty}\|B(n)\|^{2}\right)^{1 / 2}=\frac{1}{\sqrt{2 \pi}} \sum_{n=-\infty}^{\infty} \sup _{\substack{m-k=n, m, k \geqslant 1}}\left(|\hat{q}(m-k)|^{2}+|\hat{q}(m+k)|^{2}+\right. & \\
& \left.+|\hat{q}(-(m+k))|^{2}+|\hat{q}(-(m-k))|^{2}\right)^{1 / 2} \leqslant \frac{2 \sqrt{2}}{\sqrt{\pi}}\|q\| .
\end{aligned}
$$

Из полученных оценок следует, что оператор $B$ принадлежит допустимому пространству возмущений $\mathfrak{A}$, которое строится по системе ортопроекторов $\left(P_{n}\right)$. 
Для собственных значений рассматриваемого оператора $A$ имеем $(n+1)^{2}-n^{2}=2 n+1 \rightarrow \infty$ при $n \rightarrow \infty$, т.е. выполнено соотношение (3), а значит, и условия (1) и (2) на последовательность $\alpha_{m}(n)$. Кроме того, последовательность $\alpha_{m}(n)$ имеет вид $\alpha_{m}(n)=\left(m^{2}-n^{2}\right)^{-1}$. Тогда

$$
\left\|\alpha_{m}\right\|_{l_{2}(\mathbb{Z})}^{2}=\sum_{n \neq m}\left(m^{2}-n^{2}\right)^{-1}=\frac{\pi^{2}}{4 m^{2}}-\frac{3}{8 m^{4}}
$$

и, следовательно, $\left\|\alpha_{m}\right\|_{l_{2}(\mathbb{Z})} \leqslant \pi /(2 m)$. Поэтому выполняются условия теоремы 3.2 , а следовательно, и теоремы 3.3. Спектр оператора $A-B=-d^{2} / d t^{2}-q$ может быть представлен в виде

$$
\sigma\left(-\frac{d^{2}}{d t^{2}}-q\right)=\left\{\widetilde{\lambda}_{0}, \widetilde{\lambda}_{1}, \ldots, \widetilde{\lambda}_{2 m_{0}}\right\} \cup\left\{\widetilde{\lambda}_{ \pm n}, n \geqslant m_{0}+1\right\}
$$

Найдем асимптотику этого спектра. Для получения оценок собственных значений оператора $A-B$ представим подобный ему оператор $A-J_{m_{0}} X_{0}$ в виде (14); при этом

$$
P_{n} B P_{n} x=\frac{1}{\sqrt{2 \pi}}\left(\left(x, e_{n}\right) \hat{q}(0)+\left(x, e_{-n}\right) \hat{q}(2 n)\right) e_{n}+\frac{1}{\sqrt{2 \pi}}\left(\left(x, e_{n}\right) \hat{q}(-2 n)+\left(x, e_{-n}\right) \hat{q}(0)\right) e_{-n},
$$

где

$$
\hat{q}(0)=\frac{1}{\sqrt{2 \pi}} \int_{0}^{2 \pi} q(t) d t .
$$

Таким образом, оператору $\left.\left(n^{2} P_{n}-P_{n} B\right)\right|_{H_{n}}$ отвечает в базисе $e_{n} e_{-n}$ пространства $H_{n}$ матрица вида

$$
\left(\begin{array}{cc}
n^{2}-\frac{1}{\sqrt{2 \pi}} \hat{q}(0) & -\frac{1}{\sqrt{2 \pi}} \hat{q}(-2 n) \\
-\frac{1}{\sqrt{2 \pi}} \hat{q}(2 n) & n^{2}-\frac{1}{\sqrt{2 \pi}} \hat{q}(0)
\end{array}\right), \quad n>m_{0} .
$$

Ее спектр $\widetilde{\sigma}_{n}^{0}=\sigma\left(\left.\left(n^{2} P_{n}-P_{n} B\right)\right|_{H_{n}}\right)$ имеет вид

$$
n^{2}-\frac{1}{\sqrt{2 \pi}} \hat{q}(0) \pm \sqrt{\frac{1}{\sqrt{2 \pi}} \hat{q}(2 n) \hat{q}(-2 n)} .
$$

Имеют место следующие оценки:

$$
\left|\widetilde{\lambda}_{ \pm n}-n^{2}+\frac{1}{\sqrt{2 \pi}} \hat{q}(0) \pm \sqrt{\frac{1}{\sqrt{2 \pi}} \hat{q}(2 n) \hat{q}(-2 n)}\right| \leqslant 2\left\|P_{n} B\right\|_{*}\|B\|_{*}\left\|\alpha_{n}\right\|_{l_{2}(\mathbb{Z})} .
$$

Теорема 4.1. Существует такое число $m_{0}$, что при $n>m_{0}$ собственные значения дифберенииального оператора $-d^{2} / d t^{2}-q$, определяемого краевыми условиями (15), допускают следующие оченки:

$$
\left|\widetilde{\lambda}_{ \pm n}-n^{2}+\frac{1}{\sqrt{2 \pi}} \hat{q}(0) \pm \sqrt{\frac{1}{\sqrt{2 \pi}} \hat{q}(2 n) \hat{q}(-2 n)}\right| \leqslant \frac{8}{n}\|q\|^{2} .
$$

\section{СПИСОК ЛИТЕРАТУРЫ}

1. Баскаков А. Г. Гармонический анализ линейных операторов. - Воронеж: ВГУ, 1987.

2. Баскаков А. Г. Методы абстрактного гармонического анализа в теории возмущений линейных операторов// Сиб. мат. ж. - 1987. - 24, № 1. - С. 21-39.

3. Баскаков А. Г., Дербушев А. В., Щербаков А. О. Метод подобных операторов в спектральном анализе оператора Дирака с негладким потенциалом// Изв. РАН. Сер. мат. - 2011. - 75, № 3. - С. 3-28.

4. Гаркавенко Г. В. О диагонализации некоторых классов линейных операторов// Изв. вузов. Мат. 1994. - № 11. - C. 14-19.

5. Гаркавенко Г. В. Спектральный анализ возмущенных самосопряженных операторов с дискретным спектром// в кн.: Актуальные направления научных исследований XXI века: теория и практика. - Bоронеж, 2017. - C. 101-104. 
6. Гаркавенко Г. В., Ускова Н. Б. Метод подобных операторов в исследовании спектральных свойств разностного оператора с растущим потенциалом// Сиб. электр. мат. изв. - 2017. - 14. - С. 673-689.

7. Гаркавенко Г. В., Ускова Н. Б. Спектральный анализ одного класса разностных операторов с растущим потенциалом// Изв. Саратов. ун-та. Сер. Мат. Мех. Информ. - 2016. - 16, № 4. - С. 395-402.

8. Garkavenko G. V., Zgolich A. R., Uskova N. B. Spectral analysis of a difference operator with a growing potential// J. Phys. Conf. Ser. — 2018. - 973, № 1. — 012053.

Гаркавенко Галина Валериевна

Воронежский государственный педагогический университет

E-mail: g.garkavenko@mail.ru 